Bundesgesundheitsbl -

Gesundheitsforsch - Gesundheitsschutz

1999 -42: 206-211 (c) Springer-Verlag 1999

Originalien und Übersichtsarbeiten

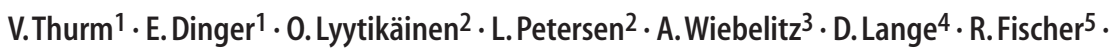
H. Oppermann ${ }^{5} \cdot$ D. Mäde ${ }^{6}$

${ }^{1}$ Bundesinstitut für gesundheitlichen Verbraucherschutz und Veterinärmedizin, Bereich Wernigerode ${ }^{2}$ Robert Koch-Institut, Berlin ${ }^{3}$ Gesundheitsamt des Landkreises Anhalt-Zerbst, Zerbst • ${ }^{4}$ Veterinär- und Lebensmittelüberwachungsamt des Landkreises Anhalt-Zerbst, Roßlau $•{ }^{5}$ Hygieneinstitut Sachsen-Anhalt, Magdeburg • 6 Veterinär- und Lebensmitteluntersuchungsamt, Halle

\title{
Infektionsepidemiologie lebensmittelbedingter Campylobacter-Infektionen
}

\section{Untersuchung eines Ausbruchs in Sachsen- Anhalt mittels epidemiologischer, mikrobiolo- gischer und molekularbiologischer Methoden}

ten Geflügelfleisch isolierten C. jejuni-Stämme zeigten gegenüber den Patientenstämmen deutlich unterscheidbare Feintypisiermuster, so daß das Geflügelfleisch als Infektionsquelle ausgeschlossen werden konnte. Die Untersuchungen unterstreichen die Nützlichkeit interdisziplinären Zusammenwirkens von humanmedizinischen und lebensmittelhygienischen Ermittlungen durch Sachverständige der Human- und Veterinärmedizin und die Aussagefähigkeit spezialisierter infektions-epidemiologischer Laboruntersuchungen bei der Ursachenermittlung lebensmittelbedingter Erkrankungen.

\section{Schlüsselwörter}

Campylobacter jejuni - Lebensmittelinfektion · Infektionsepidemiologie · Rohmilch $\cdot$ Molekularbiologische Subtypisierung
Lebensmittelinfektionen durch thermophile Campylobacter-Spezies sind wie viele andere Lebensmittelinfektionen auch weltweit im Ansteigen begriffen [1-3]. Das trifft auch auf die Bundesrepublik Deutschland zu [4-6].

Campylobacter steht als bakterieller Enteritiserreger bei Lebensmittelinfektionen in der Bundesrepublik Deutschland nach den Salmonellen an zweiter Stelle [6-8].

In einer Reihe europäischer (Großbritannien, Holland, Dänemark u.a.), aber auch außereuropäischer Länder (USA, Kanada, Südamerika, Australien) nimmt die Spezies dagegen bereits den ersten Platz der bakteriellen Erregerskala bei Enteritiden ein. Auch in der Bundesrepublik Deutschland ist dieser Trend steigend. So wurden im I. Quartal 1998 in vier Bundesländern bereits häufiger Campylobacter spp. als Salmonellen isoliert [9]. Trotzdem erscheinen Campylobacterinfektionen im gesund-

C. jejuni-Stämme vom Rind, aus Rohmilch und von den Erkrankten. Offensichtlich unzureichend in der Küche erhitzte kontaminierte Rohmilch als Zusatz zu einer Quarkspeise wurde dadurch als Infektionsquelle ermittelt. Die aus dem gleichfalls verdächtig-

Dr.V.Thurm

Robert-Koch-Institut, Bereich Wernigerode, Burgstraße 37, D-38855 Wernigerode 
V.Thurm · E. Dinger $\cdot 0$. Lyytikäinen · L. Petersen · A. Wiebelitz · D. Lange . R. Fischer $\cdot$ H. Oppermann $\cdot$ D. Mäde

Epidemiology of food-borne illnesses caused by campylobacter infections

\section{Summary}

A report is presented on an outbreak of acute gastrointestinal infections caused by Campylobacter (C.) jejuni. It occurred in SaxonyAnhalt, with a total of 186 cases in 6 kindergartens of a single township. All cases had eaten food from a common source (catering establishment). An investigation into the causes was conducted which involved specific interviews among those who had consumed the meals provided, a statistical evaluation as part of a cohort study and investigations into the conditions of food hygiene and food technology at the level of food preparation. In addition, stool specimens from cases, obligatory samples of the prepared foods, samples of the foods of animal origin used and from animals at the farm of origin were subjected to microbiological examination.Comparative subtyping of the isolates by means of molecular-biological methods demonstrated a clonal identity of the C. jejuni strains from cattle, raw milk and cases. Obviously, contaminated raw milk that had been insufficiently heated in the kitchen and added to a quark dish was found to be the source of infection. The $C$.jejuni strains isolated from poultry meat that has also been incriminated exhibited fine typing patterns that were clearly distinguishable from those of the patient strains, so that poultry meat could be excluded as source of infection. These investigations have underlined the expediency of interdisciplinary cooperation between the investigational activities of experts in medicine and the value of the evidence provided by specialized laboratory studies when having to establish the causes of food-borne illnesses.

\section{Key words}

Campylobacter jejuni • Food-borne illness • Raw milk - Molecular typing patterns heitspolitischen Interesse der Öffentlichkeit in Deutschland unterrepräsentiert. Die Gründe dafür liegen einmal im Meldesystem nach dem geltenden Bundesseuchengesetz, das eine erregerspezifische Erfassung innerhalb der Meldekategorie „übrige Formen der Enteritis infectiosa" nicht vorgibt. Weitere Gründe sind in der nicht einfachen bakteriologischen Diagnostik sowie in der bislang unzureichenden Aufklärung der Öffentlichkeit darüber zu suchen.

\section{„Campylobacter steht als bakterieller Enteritiserreger bei Lebensmittel- infektionen in der Bundesrepublik Deutschland nach den Salmonellen an zweiter Stelle [6-8]."}

Thermophile Campylobacterspezies, insbesondere Campylobacter (C.) jejuni und $C$. coli, kommen bei gesunden Säugetieren (z.B. Rindern) und Vögeln (z.B. Hühnern) als normale Besiedler des Darmes vor. Sie können durch Ausscheidung über den Kot und/oder Kontamination während des Schlachtprozesses, durch den Melkvorgang sowie durch küchentechnische Fehler in verzehrsfertige Lebensmittel gelangen. Vom Menschen werden sie vorwiegend über kontaminierte rohe oder unvollständig erhitzte tierische Lebensmittel aufgenommen. Kontaktinfektionen spielen nur bei Kindern eine Rolle. Daher ist die Campylobacteriose weitestgehend als Lebensmittelinfektion zoonotischen Ursprungs einzuordnen. Bei sporadischen Erkrankungen wird kontaminiertes Geflügel als häufige Infektionsquelle angesehen. Bei Ausbrüchen wurden der Verzehr von Rohmilch sowie das Trinken von Oberflächenwasser in der Literatur genannt [10-12]. In einzelnen Fällen kann der Erreger auch durch Kontakt mit Wildvögeln und Haustieren wie Hunden und Katzen auf den Menschen übertragen werden. Unbeschadet dieser Erkenntnisse sind noch viele Fragen zu Infektionsquellen und -wegen offen und bedürfen weiterer infektionsepidemiologischer Forschung. Anliegen dieses Artikels ist es, anhand eines konkreten Fallbeispiels Wege dazu aufzuzeigen.

\section{Falldarstellung}

Am 14.4.1997 wurde dem Gesundheitsamt in Zerbst (Sachsen-Anhalt) das explosionsartige Auftreten von Durchfallserkrankungen zeitgleich in mehreren Kindergärten der Stadt gemeldet. Als einziger Erreger wurde aus dem Stuhl der Betroffenen Campylobacter jejuni isoliert. Alle Erkrankten hatten Mittagsmahlzeiten aus einer Großküche des Landkreises Zerbst verzehrt. Die Untersuchung des Ausbruchs erfolgte zunächst durch Zusammenarbeit des Gesundheitsamts und des Veterinär- und Lebensmittelüberwachungsamtes des Landkreises Anhalt-Zerbst mit dem Hygieneinstitut Sachsen-Anhalt (Magdeburg) und dem Veterinär- und Lebensmitteluntersuchungsamt (Halle). Wegen der großen Anzahl der Erkrankungen, der Schwierigkeit des Erregernachweises in Lebensmitteln und der komplexen Vielfalt möglicher Infektionsquellen wurden zudem die Arbeitsgruppe „Aufsuchende Epidemiologie" des Robert Koch-Instituts (RKI) Berlin und die Arbeitsgruppe „Epidemiologie der Lebensmittelinfektionen" des Bundesinstituts für gesundheitlichen Verbraucherschutz und Veterinärmedizin (BgVV) um Mitarbeit gebeten.

\section{Zielsetzung}

Ziel der gemeinsamen Untersuchungen war es, mittels epidemiologischer und labordiagnostischer Methoden die Infektionsquelle zu ermitteln, den Übertragungweg zu rekonstruieren und daraus sowohl Maßnahmen im speziellen Fall wie auch allgemeine Empfehlungen für die Ursachenermittlung von Lebensmittelinfektionen und die Prävention von Campylobacter-Erkrankungen generell abzuleiten.

\section{Material und Methoden}

\section{Epidemiologie}

Die Befragungen bei den Betroffenen hinsichtlich Auftreten, Schwere und Dauer der Erkrankungen sowie zur Einnahme gemeinsamer Mahlzeiten während der für Campylobacteriosen übli- 
Tabelle 1

Speisenplan für das Kindergartenessen 07.04.-11.04.1997

Wochentag Datum Speisenkomponenten

Montag 07.04. Grüne Bohnensuppe, Mandelpudding, Schokosoße

Dienstag 08.04. Fischlies, Kräutersoße, Kartoffelbrei, Kompott

Mittwoch 09.04. Frikassee, Risotto, Fruchtjoghurt

Donnerstag 10.04. Linsensuppe, Rotwurst, Quarkspeise

Freitag 11.04. Bratklops, Kohlrabigemüse, Kartoffeln, Obst

chen Inkubationszeit wurden vom Gesundheitsamt Zerbst durchgeführt. Diese Befragungen wurden ergänzt durch eine retrospektive Kohortenstudie. Sie wurde von der Arbeitsgruppe „Aufsuchende Epidemiologie" des RKI mit Unterstützung des Hygieneinstituts Sachsen-Anhalt und des Gesundheitsamtes Zerbst durchgeführt. Dazu wurden zunächst Erhebungen mittels spezieller Fragebögen bei drei ausgewählten Personengruppen durchgeführt: Eltern der betroffenen Kinder, Mitarbeiter der Kindergärten und weitere Essenteilnehmer (Privatpersonen). Um den wahrscheinlichsten Tag der Exposition zu ermitteln, wurden die Erkrankungsraten der Essenteilnehmer, die an einem bestimmten Tag Speisen eingenommen hatten, mit den Erkrankungsraten derer, die nicht gegessen hatten, verglichen. Die für die Auswahl vorgegebene Falldefinition verlangte, daß eines oder mehrere der folgenden Merkmale von den jeweiligen Personen erfüllt wurden:

D Durchfall (d.h. drei oder mehr ungeformte Stühle pro Tag für mindestens einen Tag),

D drei oder mehr der folgenden Symptome: Erbrechen, Übelkeit, krampfartige Leibschmerzen, Fieber,

- Nachweis von Campylobacter spp. im Stuhl.

Die lebensmittelhygienischen und technologischen Ermittlungen in der Großküche, einer Fleischerei und im Milchviehbestand eines landwirtschaftlichen Betriebes wurden vom Veterinärund Lebensmittelüberwachungsamt (VLMÜA) Zerbst unter Beteiligung des BgVV durchgeführt.

\section{Mikrobiologie}

Die Untersuchung der Stuhlproben von 186 Erkrankten zum Nachweis von Enteritiserregern erfolgte in einer privaten Laborpraxis sowie im Hygieneinstitut Sachsen-Anhalt (Magdeburg). 40 der dabei erhaltenen Campylobacter-Isolate wurden dem BgVV Wernigerode zur weiteren Typisierung übersandt, zusätzlich noch fünf Isolate aus damit epidemiologisch nicht im Zusammenhang stehenden Einzelerkrankungen für Vergleichszwecke. Die Rückstellprobe des am 11.4. ausgegebenen Essens wurde im Veterinär- und Lebensmitteluntersuchungsamt Halle u.a. auf das Vorkommen von C. jejuni kulturell und durch Polymerasekettenreaktion (PCR) mittels für C. jejuni und C. coli spezifischer Primer untersucht. Des weiteren wurden zwei Geflügelfleischproben, 128 Rohmilchproben (Sammelproben und Einzelgemelke) sowie Kotproben von jeder der 98 Kühe der die Küche beliefernden Milchviehanlage durch das VLMÜA Zerbst gemeinsam mit dem BgVV Wernigerode entnommen und dort bakteriologisch untersucht. Die Anzucht und Identifizierung von Campylobacter $s p p$. aus Stuhl und Rinder-Kotproben erfolgte nach den Empfehlungen der Deutschen Gesellschaft für Hygiene und Mikrobiologie [13], aus Milchproben in Anlehnung an den ISO-Standard [14].

\section{Charakterisierung der C. jejuni-Isolate}

Die Resistenz gegenüber Nalidixinsäure, Erythromycin, Tetracyclin, Kanamycin, Streptomycin und Gentamicin wurde mittels Agardiffusionstest bestimmt [15]. Die weitere Charakterisierung der C. jejuni-Isolate (Feintypisierung) er- folgte mittels Ganzzellproteinmusterund Multilocusenzymelektrophorese (MLEE) [16], DNA-Fingerprinting mittels Pulsfeldgelelektrophorese (PFGE) [17] unter Verwendung von SmaI als Restriktionsenzym und Polymerasekettenreaktion (RAPD-PCR) [18].

\section{Ergebnisse}

\section{Epidemiologische und lebensmittel- hygienische Ermittlungen}

Nach den Ermittlungsergebnissen der Gesundheitsbehörden erkrankten vom 11. bis 23.4.1997 186 (45,1\%) von insgesamt 412 Essenteilnehmern (vorwiegend Kinder und Erzieher in sechs Kindergärten) an Durchfall mit Bauchschmerzen und z.T. hohem Fieber. Der Durchfall war bei $16 \%$ der Kinder mit Blut vermischt. Drei Kinder wurden zur stationären Behandlung in ein Krankenhaus eingewiesen. Alle Erkrankten hatten an der Gemeinschaftsverpflegung, die von einer Großküche geliefert wurde, teilgenommen. Nach vorangegangenen Erkrankungen von zwei Kindern am Freitag, dem 11.4.1997, begann der eigentliche Ausbruch am Samstag, dem 12.4.1997, und erreichte seinen Höhepunkt am darauffolgenden Montag. Deshalb wurde zunächst die Mittagsmahlzeit vom Freitag (Bratklops, Kartoffeln und Kohlrabigemüse) als Infektionsquelle angesehen (Tabelle 1). Die Untersuchung der Rückstellprobe im Veterinär- und Lebensmitteluntersuchungsamt Halle auf Enteritiserreger verlief jedoch negativ.

In Auswertung der Kohortenstudie erfüllten von 424 befragten Personen 160 die Falldefinition (s. Material und Methoden). Die statistische Analyse der Daten ergab ein erhöhtes Erkrankungsrisiko für die Essenteilnahme am Dienstag (8.4.1997) und Donnerstag (10.4.1997), hier speziell für die Speisenkomponenten Linsensuppe und Rotwurst. Aufgrund der gemeinsam vom VLMÜA und BgVV durchgeführten lebensmittelhygienischen Ermittlungen in der Gemeinschaftsküche und der angeschlossenen Fleisch- und Wurstproduktion waren jedoch sowohl die Linsensuppe wie auch die Rotwurst als Infekti- 
onsquelle praktisch auszuschließen. Berücksichtigung fanden dabei insbesondere die ermittelten technologischen Parameter (Zeit/-Temperaturverlauf der Erhitzungsprozesse) und die erregerspezifischen Besonderheiten (Tenazität) von Campylobacter jejuni.

Zum Anrühren der Quarkspeise jedoch war nach den Ermittlungsergebnissen des VLMÜ Rohmilch aus einer nahegelegenen Milchviehanlage verwendet worden, die in der Küche „aufgekocht" wurde. Während eine nachträgliche (sekundäre) Kontamination verzehrsfertiger Speisen durch infiziertes Küchenpersonal in Anbetracht negativer Stuhlbefunde unwahrscheinlich war, schlossen die lebensmittelhygienischen Ermittlungen weiterhin eine Kreuzkontamination anderer Speisen während der Zubereitung frischen Geflügels für das am Vortage (9.4.1997) ausgegebene Hühnerfrikassee nicht völlig aus. Sie wurde jedoch aufgrund des Arbeitsablaufs und der örtlichen Trennung in der Küche als eher unwahrscheinlich beurteilt.

Da Rückstellproben der ausgegebenen Speisen nicht mehr verfügbar waren, wurden zur Klärung mittels labordiagnostischer Methoden die o.g. Proben (s. Material und Methoden) entnommen und mikrobiologisch untersucht.

\section{Mikrobiologische Untersuchungen}

Bei 115 (61,8\%) der 186 Erkrankten wurde $C$. jejuni als einziger Enteritiserreger aus dem Stuhl isoliert. Bei den übrigen Stuhlproben verlief die Untersuchung negativ. In der Rückstellprobe der Gemeinschaftsverpflegung vom 11.4. wurden pathogene Keime nicht nachgewiesen. Aus einer der beiden Geflügelfleischproben, die noch tiefgefroren vorrätig lagen, wurden zwei C. jejuni-Stämme angezüchtet. Aus den 128 Rohmilchund 98 Rinderkotproben konnte in vier bzw. fünf Fällen $C$. jejuni isoliert werden.

\section{Feintypisierung der Isolate}

Alle 40 untersuchten $C$. jejuni-Isolate von Erkrankten waren sensibel gegenüber Erythromycin, Nalidixinsäure, Tetracyclin, Streptomycin, Kanamycin und Gentamicin. Sie zeigten sowohl im Ganzzellproteinprofil (Abb. 1) als auch in der MLEE und PFGE (Abb. 2) ein einheitliches Muster. Davon schon im Resistenzmuster deutlich unterscheidbar war eines der beiden Isolate aus Geflügelfleisch durch Resistenz gegenüber Nalidixinsäure und Tetracyclin (Tabelle 2). Auch anhand der Muster ihrer Ganzzellproteine, der Alleloenzyme (Malic enzyme und Isocitratdehydrogenase) sowie mittels Pulsfeldgelelektrophorese (Abb. 2) erwiesen sie sich untereinander als zwei verschiedene Stämme, die sich andererseits beide deutlich von den Patientenisolaten des Ausbruchs unterschieden (Tabelle 2). Dagegen wurden aus drei von vier Rohmilchproben C. jejuni-Stämme angezüchtet, die sich in der Feintypisierung mittels der o.g. Methoden als mit dem Ausbruchsstamm identisch erwiesen. Auch die mit ausgewählten Stämmen durchgeführte RAPD bestätigte eindeutig dieses Ergebnis. Die gleichen Muster fanden wir auch bei drei der fünf $C$. jejuni-Isolate aus Rinderkot. Zwei Rinderkot- und ein Rohmilchstamm zeigten jeweils andere Muster.

Tabelle 2

Epidemiologische Feintypisierung ausgewählter C. jejuni-Isolate

\begin{tabular}{|c|c|c|c|c|c|c|c|}
\hline \multirow[t]{2}{*}{ Material } & \multirow[t]{2}{*}{ Anzahl der Isolate } & \multirow[t]{2}{*}{ Resistenzmuster } & \multirow[t]{2}{*}{ Ganzzellproteinmuster } & \multicolumn{2}{|c|}{ Enzymmuster } & \multicolumn{2}{|c|}{ DNA-Fingerprinting } \\
\hline & & & & ME & ICD & PFGE & RAPD \\
\hline $\begin{array}{l}\text { Stuhlproben } \\
\text { Ausbruch }\end{array}$ & 40 & sens & 2.8 .0 & 4 & 1 & A & A \\
\hline $\begin{array}{l}\text { Stuhlprobe } \\
\text { Einzelerkrankung }\end{array}$ & 1 & sens & 3.1 .0 & 6 & 2 & H & - \\
\hline $\begin{array}{l}\text { Stuhlprobe } \\
\text { Einzelerkrankung }\end{array}$ & 1 & NAL & 2.5 .0 & 2 & 1 & K & - \\
\hline Rohmilch & 3 & sens & 2.8 .0 & 4 & 1 & A & A \\
\hline Rohmilch & 1 & sens & 2.8.1 & 4 & 1 & B & B \\
\hline Rinderkot & 3 & sens & 2.8 .0 & 4 & 1 & A & A \\
\hline Rinderkot & 1 & NAL, ERY & 2.8.0 & 4 & 1 & A & A \\
\hline Rinderkot & 1 & sens & 2.8.1 & 4 & 2 & $\mathrm{E}$ & \\
\hline Geflügelfleisch & 1 & NAL,TET & 2.8.2 & 2 & 3 & c & - \\
\hline Geflügelfleisch & 1 & sens & 2.8.3 & 5 & 1,5 & D & - \\
\hline
\end{tabular}

sens=sensibel; $N A L, E R Y, T E T=$ resistent gegenüber Nalidixinsäure, Erythromycin bzw. Tetracyclin; $M E=$ malic enzyme, ICD=Isocitratdehydrogenase; $P F G E=$ pulsed field gel electrophoresis, $R A P D=$ random amplified polymorphic $D N A$ analysis.

Die Buchstaben ( $A, B, C$ usw.) beim DNA-Fingerprinting charakterisieren jeweils ein bestimmtes elektrophoretisches Bandenmuster in der PFGE und RAPD (s. Material und Methoden). Die Enzymmuster sind mit Zahlen (z.B. Typ 4) in Abhängigkeit von der elektrophoretischen Mobilität der betr. Alleloenzyme (ME, ICD) dargestellt. Der Zahlencode der Ganzzellproteinmuster charakterisiert auf der Basis eines von den Autoren entwickelten Systems (16) Anzahl, Lage und ggf. Intensität von mayor- und minor-Proteinen 


\section{Diskussion}

Nach heutigem wissenschaftlichem Erkenntnisstand ist von einer klonalen Identität der untersuchten Campylobacter-Stämme auszugehen, wenn sich nach komplexer Auswertung der mittels verschiedener Methoden erhaltenen Typisiermuster übereinstimmende Aussagen ergeben [19]. Genetischer Hintergrund der Anwendung dieser Methoden für epidemiologische Zwecke ist einmal die klonale Vielfalt (Polyklonalität) der das Infektionsgeschehen beherrschenden sowie in Tieren und Lebensmitteln vorkommenden Campylobacter-Stämme. Sie läßt eine relativ sichere Unterscheidung (genetic diversity) epidemiologisch nicht relevanter Isolate (unrelated strains) zu, wie die erhaltenen Ergebnisse unterstreichen.

\section{„Die Campylobacter-Subtypisierung gestattet es, in einem begrenzten geographischen und zeitlichen Rah- men gewonnene Isolate unterschied- licher Herkunft bei identischen Typi- siermustern als gemeinsamen, klona- len Ursprungs zu betrachten [20, 21]."}

Mittels Subtypisierung unterscheidbare Stämme können auf dieser Basis als epidemiologisch nicht relevant beurteilt werden. Für die Interpretation der einheitlichen Typisiermuster der 40 von uns untersuchten Patientenisolate aus den Kindergärten läßt diese klonale Identität auf eine für einen Ausbruch charakteristische gemeinsame Infektionsquelle schließen. Dies steht im Einklang mit dem Ergebnis der epidemiologischen Ermittlungen (explosionsartiger Ausbruch der Erkrankungen). Mehrere in der gleichen Region aus Einzelerkrankungen ohne epidemiologischen Zusammenhang mit dem Ausbruch isolierte Campylobacterstämme (Nr. 6 in Abb.1) erwiesen sich bereits im Ganzzellproteinprofil als davon deutlich different. Das unterstreicht die auch schon in früheren Untersuchungen [22-24] von uns festgestellte diskriminierende Fähigkeit und Eignung dieser Methode zur epidemiologischen Subty- pisierung bei C. jejuni. Auch Vandamme u.a. [25] fanden verschiedene elektrophoretische Proteinmuster innerhalb einer Campylobacter-Spezies.

Da sich die beiden C. jejuni-Stämme aus dem Geflügelfleisch deutlich vom Ausbruchsstamm unterschieden (Abb. 2), konnte dieses Lebensmittel als Infektionsquelle - auch über eine evtl. Kreuzkontamination mit anderen Speisen - ausgeschlossen werden. Dies wiederum steht im Einklang mit dem Ergebnis der lebensmittelhygienischen Ermittlungen (s.o.), die aufgrund der örtlichen Gegebenheiten innerhalb der Küche eine hiervon ausgehende indirekte Übertragung über sekundär kontaminierte Lebensmittel als sehr unwahrscheinlich erscheinen ließen. Gegen eine direkte Infektion über das hergestellte Frikassee hatten bereits die Auswertungen der Kohortenstudie und der vorangegangene Kochprozeß eindeutig gesprochen. Dagegen ist die nachgewiesene klonale Identität von jeweils drei aus Rohmilch und drei aus Rinderkot isolierten C. jejuni-Stämmen mit dem Erregerstamm des Ausbruchs unter den ermittelten Umständen eindeutig als laboranalytischer Beweis für dessen Herkunft zu werten. Die mit C. jejuni kontaminierte Rohmilch aus der die Großküche beliefernden Milchviehanlage ist somit als Überträger (Vehikel) des infektiösen Agens anzusehen.

Dieser laboranalytisch mittels genetischen Fingerprints geführte Nachweis

Abb. 1 $\Delta$ Ganzzellproteinprofil von C.-Isolaten. St=Molekulargew.-Standard; 1-5=PatientenIsolate Ausbruch; $6=$ =Isolat aus Einzelerkrankung anderer Herkunft; 7-9=Rohmilch-Isolate; 10-11=Rinderkot-Isolate zeigt die zoonotische Infektkette von den mit Campylobacter besiedelten Milchkühen via Kot und Rohmilch in die Gemeinschaftsküche und von dort über die damit angerührte Quarkspeise zu den Erkrankten auf. Die Kohortenstudie hatte das höchste Infektionsrisiko für das Mittagessen des 10.4.1997 herausgestellt. Das steht miteinander im Einklang.

\section{Schlußfolgerungen}

In Auswertung aller vorliegenden Untersuchungsergebnisse lassen sich folgende Schlußfolgerungen ableiten:

1. Der Verzehr roher bzw. unzureichend erhitzter Milch ist ein nicht zu unterschätzendes Risiko für lebensmittelbedingte Campylobacter-Infektionen.

2. Die sinnvolle Kombination zielgerichteter epidemiologischer und lebensmittelhygienischer Ermittlungen bei Patienten und im Bereich der Speisenproduktion mit laboranalytischen Ergebnissen erweist sich als eine geeignete Methode für die Untersuchung von Campylobacter-Ausbrüchen, übertragbar auch auf andere Lebensmittelinfektionen.

3. Mittels molekularer Charakterisierung von Campylobacter-Isolaten aus gezielt entnommenen Proben ist es möglich, mutmaßliche Infektionsquellen und -wege zu verifizieren und infektionsepidemiologische Schlußfolgerungen für die Bekämpfung und

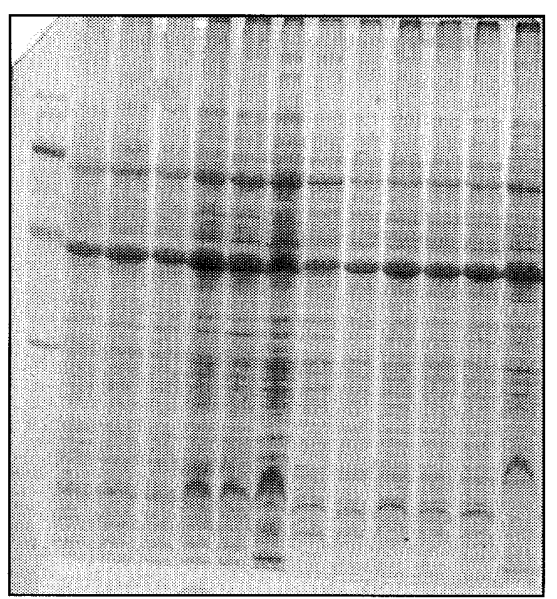

$\begin{array}{lllllllllllll}\text { St } 1 & 2 & 3 & 4 & 5 & 6 & 7 & 8 & 9 & 10 & 11 & 12\end{array}$ 


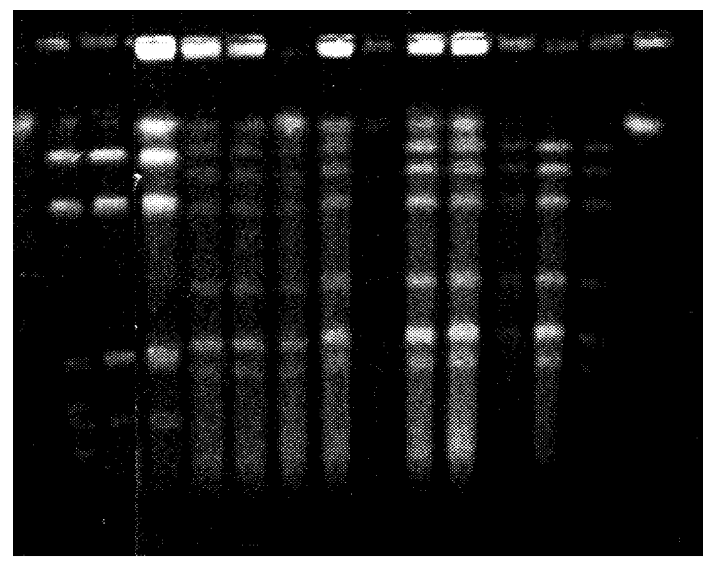

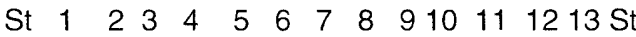

Abb. $2 \triangle$ DNA Fingerprinting (PFGE) von C. jejuni-Isolaten. 1-3=Geflügelfleisch-Isolate; 4, $5=$ Rohmilch-Isolate; $6-10=$ Rinderkot-Isolate; 11-13=PatientenIsolate Ausbruch; $S t=$ Molekulargew.-Standard
Prophylaxe lebensmittelbedingter Campylobacteriosen abzuleiten.

Optimale Ergebnisse lassen sich bei Ausbruchsuntersuchungen mit Verdacht auf lebensmittelbedingte Infektionen nur durch interdisziplinäre Zusammenarbeit der örtlich zuständigen Behörden sowohl des Gesundheits- wie auch des Veterinärwesens unter Einbeziehung von Speziallaboratorien erzielen. Die beiden beteiligten Bundesinstitute verfügen dafür über entsprechendes wissenschaftliches und technisches Fachpersonal sowie über labordiagnostische Möglichkeiten.

Der Gesetzgeber der Bundesrepublik Deutschland hat inzwischen unter gleichzeitiger Berücksichtigung der Infektionsgefahr durch EHEC-Bakterien über rohe tierische Lebensmittel den vom BgVV vorgelegten Forschungsergebnissen der letzten Jahre zur Infektionsgefährdung durch Campylobacter über Rohmilch Rechnung getragen [26]. Die Novellierung des $₫ 18$ der Milchverordnung verbietet nunmehr generell die Abgabe von Rohmilch einschließlich Vorzugsmilch ohne ausreichende Erhitzung in Gemeinschaftsküchen an Verbraucher. Damit wurde eine wesentliche Verbesserung des gesundheitlichen Verbraucherschutzes in diesem Bereich erzielt.

Der Laborarztpraxis Dr. Lorenz in Dessau danken wir für die Überlassung von Campylobacter-Isolaten dieses Ausbruchs. Für die Durchführung der epidemiologischen Ermittlungen in den
Kindergärten danken wir Frau Krause, Frau Neumann und Frau Knape vom Gesundheitsamt Zerbst, für die gewissenhaften mikrobiologischen und molekularbiologischen Untersuchungen unseren MTA Frau Geringer, Frau Stöckel und Frau Friedrich.

\section{Literatur}

1. Healing TD, Greenwood MH, Pearson AD (1992) Campylobacters and enteritis. Reviews in Med Microbiol 3:159-167

2. Phillips CA (1995) Incidence, epidemiology and prevention of foodborne Campylobacter species. Trends in Food Science \& Technology 6:83-87

3. Käferstein F,Abdussalam M (WHO) Food safety in the 21. Century. Vortrag 4th World Congress Foodborne Infections and Intoxications, Berlin, 7.-12.June 1998

4. Rasch G, Apitzsch L, Menzel U (1996) Wichtige Entwicklungen bei meldepflichtigen bakteriellen Infektionskrankheiten im Jahr 1995. InfFo Sonderheft A:20-22

5. Epidemiologisches Bulletin des RKI 3/1997. Statistische Angaben zu ausgewählten Infektionskrankheiten 1996

6. Epidemiologisches Bulletin des RKI 8/1997.Jahresbericht 1996 über meldepflichtige Infektionskrankheiten in Deutschland. Teil 1:Darminfektionen

7. Thurm V, Dinger $E$ (1993) Epidemiologie der Lebensmittelinfektionen als laborgestützte Infektionsepidemiologie im Bereich der Veterinärmedizin. Bundesgesundhbl 36:308-313

8. Pressedienst 06/1998 des Bundesinstituts für gesundheitlichen Verbraucherschutz und Veterinärmedizin: Campylobacter jejuni - als Erreger bakterieller Lebensmittelinfektionen vielfach unterschätzt

9. Epidemiologisches Bulletin des RKI 18/1998.Quartalsstatistik und Situationsbericht: Enteritis infectiosa übrige Formen
10. Kapperud G, Skyerve E, Bean NH, Ostroff SM, Lassen J (1992) Risk factors for sporadic campylobacter infections: results of a case-control study in southeastern Norway.J Clin Microbiol 30:3117-3121

11. Wood RC, MacDonald KL, Osterholm MT (1992) Campylobacter enteritis outbreaks associated with drinking raw milk during youth activities. JAMA 268:3228-3230

12. Morgan D, Gunneberg C, Gunnell D, Healing TD, Lamerton S. Soltanpoor N, Lewis DA, White DG (1994) An outbreak of Campylobacter infection associated with the consumption of unpasteurised milk at a large festival in England. Eur J Epidemiol 10: 581-585

13. Kist $M(1991)$ Isolierung und Identifizierung von Bakterien der Gattungen Campylobacter und Helicobacter.Zbl Bakt 276:124-139

14. International Organization for Standardization (1994) Draft International Standard ISO/DIS 10272:Microbiology - General guidance for detection of thermotolerant Campylobacter

15. Gaudreau C, Gilbert H (1997)Camparison of disc diffusion and agar dilution methods for antibiotic susceptibility testing of Campylobacter jejuni subsp. jejuni and Campylobacter coli. J Antimicrob Chemother 39:707-712

16. Thurm V, Dinger E (1994) Application of subtyping by combined allozyme, whole-cell protein and antibiotic resistance analysis in epidemiological investigations of foodborne infections. Intern J Food Microbiol 24:261-271

17. Yan W, Chang N, Taylor DE (1991) Pulsed-field gel electrophoresis of Campylobacter jejuni and Campylobacter coli genomic DNA and its epidemiologic application.J Infect Dis 163: 1068-1072

18. Tichy H-V, Simon R (1994) Effiziente Analyse von Mikroorganismen mit PCR-Fingerprint-Verfahren. Bioforum 17:499-505

19. Gibson JR, Fitzgerald COwen RJ (1995) Comparison of PFGE, ribotyping and phage-typing in the epidemiological analysis of Campylobacter jejuni serotype HS2 infections. Epidemiol Infect 115:215-225

20. Wassenaar TM, Geilhausen B Newell DG (1998) Evidence of genomic instability in Campylobacter jejuni isolated from poultry. Appl Environm Microbiol 64:1816-1821

21. Thurm V et al. Genetic diversity and clonal relationships of Campylobacter jejuni in poultry flocks. Epidemiol Infect (im Druck)

22. Thurm V, Teufel P, Dinger E, Bartelt E (1994) Gemeinschaftsverpflegung als Infektionsquelle eines Campylobacter-jejuni-Ausbruchs. Jahresbericht des BgVV, S 99-100

23. Thurm V, Dinger $E$ (1996) Rohmilch in der Gemeinschaftsverpflegung als Infektionsquelle eines Campylobacter-jejuni-Ausbruchs in Niedersachsen. Jahresbericht des BgVV, S120-121

24. Thurm V, Dinger E (1998) Subtyping of outbreak-related strains as a useful method in the surveillance of Campylobacter infections. Proceedings 4. Weltkongreß Lebensmittelinfektionen und -intoxikationen Berlin 1998

25. Vandamme P, Pot B, Falsen E, Kersters K, De Ley J (1990) Intra- and interspecific relationships of veterinary campylobacters revealed by numerical analysis of electrophoretic protein profiles and DNA: DNA hybridizations. System Appl Microbiol 13: 295-303

26. Bundesgesetz.-bl. 1997 Teil I Nr.80 vom 09.12.1997 